\title{
Large-scale magnetic fields, curvature fluctuations, and the thermal history of the Universe
}

\author{
Massimo Giovannini* \\ Centro Enrico Fermi, Via Panisperna 89/A, 00184 Rome, Italy \\ and Department of Physics, Theory Division, CERN, 1211 Geneva 23, Switzerland
}

(Received 5 July 2007; published 5 November 2007)

\begin{abstract}
It is shown that gravitating magnetic fields affect the evolution of curvature perturbations in a way that is reminiscent of a pristine nonadiabatic pressure fluctuation. The gauge-invariant evolution of curvature perturbations is used to constrain the magnetic power spectrum. Depending on the essential features of the thermodynamic history of the Universe, the explicit derivation of the bound is modified. The theoretical uncertainty in the constraints on the magnetic energy spectrum is assessed by comparing the results obtained in the case of the conventional thermal history with the estimates stemming from less conventional (but phenomenologically allowed) post-inflationary evolutions.
\end{abstract}

DOI: 10.1103/PhysRevD.76.103508

\section{INTRODUCTION}

It is tempting to speculate that large-scale magnetic fields are generated in the early Universe [1-3] during a phase where the Weyl invariance of their evolution equations is broken either spontaneously or explicitly (see, for instance, [4] and references therein). As a result the obtained magnetic fields will be necessarily tangled over typical wavelengths larger than the Hubble radius [5-9]. In this situation, the presence of magnetic fields may affect the evolution of curvature inhomogeneities in a way that is reminiscent of what happens in the presence of nonadiabatic pressure fluctuations.

Suppose, indeed, that large-scale magnetic fields are generated thanks to the amplification of the quantum fluctuations of an Abelian gauge field which can be identified with the hypercharge field whose modes will not be screened by thermal effects. In spite of the specific model of parametric amplification, the result of the process will be a stochastically distributed field whose two-point function can be written as

$$
\left\langle B_{i}(\vec{k}, \tau) B^{j}(\vec{p}, \tau)\right\rangle=\frac{2 \pi^{2}}{k^{3}} P_{i}^{j}(k) P_{\mathrm{B}}(k) \delta^{(3)}(\vec{k}+\vec{p}),
$$

where $P_{\mathrm{B}}(k)$ is the magnetic power spectrum (which may change depending upon the specific way the Weyl invariance is broken) and $P_{i}^{j}(k)=\left(\delta_{i}^{j}-k_{i} k^{j} / k^{2}\right)$ is the traceless projector. In Eq. (1.1) the momenta appearing in the correlator are the comoving wave numbers. The homogeneous component of this configuration vanishes and, as a result, the magnetic fields will not break spatial isotropy ${ }^{1}$ of the background geometry whose line element can be written,

\footnotetext{
*massimo.giovannini@cern.ch

${ }^{1}$ If a background magnetic field exists, it must necessarily be oriented along a specific direction [10]. The effective spatial isotropy is broken and the angular power spectrum depends on the direction of the magnetic field. As a consequence a number of constraints, stemming directly from the breaking of spatial isotropy, can be derived [11].
}

PACS numbers: 98.80.Cq, 95.85.Sz, 98.62.En, 98.70.Vc

in the spatially flat case and in terms of the conformal time coordinate $\tau$, as

$$
d s^{2}=a^{2}(\tau)\left[d \tau^{2}-d \vec{x}^{2}\right] .
$$

The typical wavelengths that set the initial conditions of the cosmic microwave background $(\mathrm{CMB})$ anisotropies ${ }^{2}$ are still larger than the Hubble radius (i.e. $k \tau<1$ ) at recombination, taking place for an approximate redshift $z_{\text {rec }} \sim 1050$ when the visibility function is peaked. When $k \tau<1$ the evolution equations of the perturbations of the spatial curvature will receive contributions from three independent sources. The adiabatic mode typically provides the first contribution arising from the quantum fluctuations of a (single) inflaton field. The second contribution may come from a collection of nonadiabatic modes that may be present either because the inflationary evolution is driven by more than one scalar degree of freedom, or because the primordial plasma contains a number of spectator fields which do not drive the evolution of the background but whose inhomogeneities contribute to the overall fluctuations of the spatial curvature. Finally, the third contribution to the evolution of curvature fluctuations can be attributed to gravitating magnetic fields. Up to now various studies addressed the interplay between large-scale magnetic fields and the scalar [12-14] (see also [15,16]), vector [17-19], and tensor [19] modes of the geometry (see $[20,21]$ for two recent reviews). As far as the scalar modes are concerned, it was assumed that large-scale magnetic fields are present prior to recombination (but after equality) and the resulting corrections to the Sachs-Wolfe plateau have been computed $[12,13]$. The analysis has been also recently extended to cover the Doppler region, i.e. the first, second, and third peaks of the temperature autocorrelations [14]. The main theme of the present paper will be to discuss possible (further) numerical constraints arising from the coupled evolution of large-scale magnetic fields

\footnotetext{
${ }^{2}$ By this expression I mean the wavelengths that set the initial conditions of the lowest multipoles of the Boltzmann hierarchy.
} 
and curvature perturbations. This analysis, when correctly performed, allows assessment of the theoretical uncertainty of the computational scheme. In fact, the results obtained in the case of the conventional thermal history can be compared with the estimates stemming from less conventional (but phenomenologically allowed) postinflationary evolutions.

In the most simplistic ${ }^{3}$ scenario a conventional inflationary phase is followed, after a sudden reheating, by a radiation-dominated phase which is replaced, at equality, by the standard matter-dominated phase. This evolutionary history allows computation of the curvature perturbations that are induced, for instance, by a single primordial adiabatic mode. It is also possible, in the same framework, to include the peculiar effect of large-scale magnetic fields whose inhomogeneities may indeed affect the curvature perturbations $[12,13,15,16]$. The result of this calculation allows the estimate of the Sachs-Wolfe plateau and, ultimately, the estimate of the Doppler oscillations [14].

If the evolutionary history of the background geometry changes in its early phases, also the effect of large-scale magnetic fields on the induced curvature perturbations will be different. The evolution of magnetized curvature perturbations is sensitive, both to the total barotropic index and to the total sound speed, i.e.

$$
w_{\mathrm{t}}(\tau)=\frac{p_{\mathrm{t}}}{\rho_{\mathrm{t}}}, \quad c_{\mathrm{st}}^{2}(\tau)=\frac{p_{\mathrm{t}}^{\prime}}{\rho_{\mathrm{t}}^{\prime}}
$$

where the prime denotes a derivation with respect to $\tau$ and the subscript $\mathrm{t}$ is a reminder that the barotropic index and the sound speed are the ones pertaining to the total fluid.

If the rate of expansion changes in the early phases of the thermal history of the Universe (typically prior to big-bang nucleosynthesis) also $w_{\mathrm{t}}$ and $c_{\mathrm{st}}^{2}$ will be different and the curvature perturbations will have a slightly different evolution. This qualitative argument will now be corroborated

\footnotetext{
${ }^{3}$ Notice that the most simplistic scenario is also the one contemplated by the minimal paradigm compatible with the three data sets that are used to infer the values of the cosmological parameters, i.e. the $\Lambda \mathrm{CDM}$ paradigm. These three data include, in general terms, the large-scale structure observations, by the CMB observations and by the type Ia supernovae observations. Before plunging into the discussion, it is appropriate to comment on the choice of the cosmological parameters that will be employed throughout this section. The WMAP data [22-26] have been combined, so far, with various sets of data. These data sets include the 2dF Galaxy Redshift Survey [27], the combination of Boomerang and ACBAR data [28,29], and the combination of CBI and VSA data [30,31]. Furthermore the WMAP 3year data have been also combined with the Hubble Space Telescope Key Project (HSTKP) data [32] as well as with the Sloan Digital Sky Survey (SDSS) [33,34] data. Finally, the WMAP 3-year data can be also usefully combined with the weak lensing data [35,36] and with the observations of type Ia supernovae (in particular the data of the Supernova Legacy Survey (SNLS) [37] and the so-called Supernova "Gold Sample" (SNGS) [38,39]).
}

by a detailed calculation, and this is one of the themes of the present investigation. The problem then becomes to estimate the total curvature perturbation that will be sensitive both to the adiabatic and to the magnetized contributions.

The strategy adopted in the present investigation will then be to scrutinize different thermodynamic histories of the Universe and to compute, in each of these cases, the resulting curvature perturbations for wavelengths that are still larger than the Hubble radius after matter-radiation equality. It will be shown that possible variations in the evolution of the total barotropic index will modify the interplay between the adiabatic and the magnetized components of curvature perturbations. This procedure will also allow determination of the theoretical error caused by selecting the most simplistic thermal history in comparison with its nonminimal counterparts.

Before passing to the plan of the paper, it is appropriate to comment on the possible magnetogenesis mechanisms. At the moment it is not clear whether or not large-scale magnetic fields are really present prior to radiation-matter equality or if they are the result of some kind of battery operating much later. There exist extended reviews on the subject (see, for instance, [4,21]) and it would be difficult to reproduce here, in full details, the various aspects of the problem. Consider then, for simplicity, the quantity $B_{\mathrm{L}}$ which will be later defined in proper terms and which measures the magnetic field strength appropriately averaged over the comoving scale $\mathrm{L}$ at the onset of gravitational collapse. The first important piece of information is that the typical scale L should be sufficiently large (of the order of the Mpc) if I want the galactic field to be coherent over the collapsed protogalaxy. This consideration already excludes the possibility that large-scale magnetic fields are produced by various phase transitions operating inside the Hubble radius: the resulting correlation scale at the onset of gravitational collapse would be far too small even if the appropriate requirements on the strength of some hypothetical first-order phase transition would be met (see again [4,21]). What can be said about the strength of $B_{\mathrm{L}}$ ? Let me first presume that the only possible amplification of the primordial magnetic field is provided by gravitational collapse. Indeed, during gravitational collapse, thanks to the large value of the conductivity, the magnetic flux is conserved [21]. In this situation the primordial seed ${ }^{4}$ will experience an amplification that can be estimated in a factor $10^{4}$ or even $10^{5}$. This means that the seed must lie in the range $0.01 \mathrm{nG}$ if I want to reproduce the correct value of the galactic magnetic field. Always at the level of the galaxy it has been speculated (and often affirmed) that the dynamo mechanism could efficiently operate in such a way that the primordial seed could be safely in the range of $10^{-19} \mathrm{G}$ (in

\footnotetext{
${ }^{4}$ I will dub with seed the primordial value of the magnetic field present prior to the gravitational collapse of the protogalaxy.
} 
the absence of any subsequent compressional amplification) or even in the range $10^{-23} \mathrm{G}$ (if compressional amplification is taken into account). These numbers (usually quoted) are far too optimistic in the light of the present understanding of dynamo amplification [21].

Without going through the details I can say that there exist various dynamos. The common denominator is that a dynamo, by definition, is a mechanism able to convert the kinetic energy of the plasma into magnetic energy, and it stems directly from the so-called magnetic diffusivity equation which is a direct consequence of single fluid plasma descriptions such as magnetohydrodynamics. Now, the figure $10^{19} \mathrm{G}$ refers to the case when the dynamo is able to amplify the primordial seed by one e-fold for each galactic rotation. This means that, under this presumption, the total amount of amplification will be roughly 30 efolds, i.e. $10^{13}$ which allows a magnetic field, at the onset of galactic rotation, of $10^{-19} \mathrm{G}$. This figure assumes that only the large-scale modes of the fields are amplified. In recent years, however, it has been appreciated that not only the large wavelengths are amplified, but also the smaller ones [21]. This implies that the dynamo action will be quenched, i.e. the dynamo will be prematurely saturated. Saturation means that, for some wavelengths of the magnetic field (typically smaller than the kpc) the kinetic energy will be of the order of the magnetic energy and the pumping action on the scales much larger than the kpc will be stopped. The conclusion will be that, still being optimistic, the dynamo action (supplemented by compressional amplification) will be able to provide, at most, 26 effective e-folds of amplification which will bring up the putative value of the seed field at the level of $10^{-18} \mathrm{G}$ or even $10^{-16} \mathrm{G}$.

The question I want to address is now the following: Are there mechanisms that can produce large-scale magnetic fields with the features I just mentioned? The answer to this question is yes, but it is hard to claim that a mathematical possibility is a fact of nature unless direct constraints (or hopefully observations) from CMB physics will allow an evidence which is more compelling than theoretical prejudice. To produce coherent fields over scales comparable with the scale of the gravitational collapse of the protogalaxy the quantum fluctuations of the standard model hypercharge field must be amplified and this can be achieved, in several ways, by breaking the Weyl invariance of the corresponding evolution equations. The easiest possibility, as argued some time ago, is to embed the coupling of the dilaton to the gauge fields in the framework of a conventional inflationary model. This was the spirit of the exercise reported in [40] which allowed magnetic fields of the order of $10^{-12} \mathrm{G}$ or even $10^{-10} \mathrm{G}$ at the onset of gravitational collapse. Of course also smaller fields were allowed in that context but the possibility I just quoted has the advantage of not relying on the dynamo amplification. This class of models is under further scrutiny in the light of possible
CMB constraints [41]. Values of the magnetic field corresponding to fractions of the $\mathrm{nG}$ can be also obtained in less conventional scenarios where the internal dimensions are dynamical (and thus breaking naturally the aforementioned Weyl invariance) $[8,9]$ or even in the case of pre-big-bang models [7] where the growth of the gauge coupling in the pre-big-bang phase naturally produces an amplification of the quantum fluctuations of the hypercharge field. Possible constraints on the scenario can arise from Faraday rotation as argued long ago [42]. There is also the possibility that gauge fields can be directly coupled to the inflaton field [5]. This possibility is viewed as dangerous by some authors since the effective coupling constant might inherit large radiative corrections which might crucially alter the flat nature of the inflationary potential. Finally, there is also the possibility that the breaking of Weyl invariance directly stems from the explicit breaking of gauge invariance [6]. This would imply an effective mass of the photon which might be difficult to accept.

The bottom line of this reasoning is that the true problem is not, in the concerned viewpoint of the author, how to produce large-scale magnetic fields or how to enforce a specific theoretical prejudice (which can be, however, rather valuable). The problem is to understand if they are present prior to matter-radiation equality, and this is the rationale of looking, as carefully as possible, to $\mathrm{CMB}$ physics.

The plan of the present analysis will then be the following. In Sec. II the essential theoretical tools will be introduced. A consistent gauge-invariant description will allow following, in one shot, the evolution of the curvature fluctuations and of the density contrasts on comoving orthogonal hypersurfaces. In Sec. III different models for the evolution of the barotropic index will be scrutinized and motivated. The resulting (magnetized) curvature perturbations will be computed. In Sec. IV a set of bounds on the magnetic field intensity will be derived and compared. Sec. V contains the concluding remarks.

\section{EVOLUTION EQUATIONS}

The notations ubiquitously employed in the present script imply that the Friedmann-Lemaitre equations in the spatially flat case [with line element given in Eq. (1.2)] are given by

$$
\begin{gathered}
\mathcal{H}^{2}=\frac{8 \pi G}{3} a^{2} \rho_{\mathrm{t}}, \\
\mathcal{H}^{2}-\mathcal{H}^{\prime}=4 \pi G a^{2}\left(\rho_{\mathrm{t}}+p_{\mathrm{t}}\right), \\
\rho_{\mathrm{t}}^{\prime}+3 \mathcal{H}\left(\rho_{\mathrm{t}}+p_{\mathrm{t}}\right)=0,
\end{gathered}
$$

where $\mathcal{H}=a^{\prime} / a$ and the prime denotes, as in Eq. (1.3), a derivation with respect to $\tau$. Notice that $\mathcal{H}=a H$ where $H=\dot{a} / a$ is the Hubble expansion rate and the dot denotes a derivation with respect to the cosmic time coordinate $t$ 
(recall that the connection between $t$ and $\tau$ is given by the differential relation $d t=a(\tau) d \tau)$. By virtue of the connection between $\mathcal{H}$ and $H$, a given wavelength is larger than the Hubble radius provided the corresponding comoving wave number $k$ satisfies the condition $k /(a H) \simeq k \tau<1$. Eqs. (1.3) and (2.3) imply

$$
c_{\mathrm{st}}^{2}=w_{\mathrm{t}}-\frac{w_{\mathrm{t}}^{\prime}}{3 \mathcal{H}\left(w_{\mathrm{t}}+1\right)}=w_{\mathrm{t}}-\frac{1}{3} \frac{d \ln \left(w_{\mathrm{t}}+1\right)}{d \ln a},
$$

so that $c_{\mathrm{st}}^{2}=w_{\mathrm{t}}$ iff the (total) barotropic index is constant in time. For the purposes of the present investigation it is practical to start with the evolution equations of the fluctuations of the geometry expressed in fully gauge-invariant terms. The Hamiltonian and momentum constraints can be written as

$$
\begin{gathered}
\nabla^{2} \Psi-3 \mathcal{H}\left(\mathcal{H} \Phi+\Psi^{\prime}\right)=4 \pi G a^{2}\left(\delta \rho_{\mathrm{t}}+\delta \rho_{\mathrm{B}}\right), \quad(2.5) \\
\nabla^{2}\left(\mathcal{H} \Phi+\Psi^{\prime}\right)=-4 \pi G a^{2}\left(1+w_{\mathrm{t}}\right) \rho_{\mathrm{t}} \theta_{\mathrm{t}}, \quad \theta_{\mathrm{t}}=\nabla^{2} V_{\mathrm{t}},
\end{gathered}
$$

where $\delta \rho_{\mathrm{t}}$ is the total (and gauge-invariant) density contrast of the fluid sources and $\delta \rho_{\mathrm{B}}(\tau, \vec{x})=B^{2}(\vec{x}) /\left(8 \pi a^{4}\right)$. The fluctuations $\Phi$ and $\Psi$ are the gauge-invariant Bardeen potentials $[43,44]$ that coincide with the longitudinal fluctuations of the metric in the conformally Newtonian gauge. In Eq. (2.6) $\theta_{\mathrm{t}}$ is the three-divergence of the total peculiar velocity. At the right-hand side of Eq. (2.6) the threedivergence of the Poynting vector leads to a term going as $\vec{\nabla} \cdot[\vec{E} \times \vec{B}] / a^{4}$, where $\vec{E} \simeq \vec{J} / \sigma \simeq(\vec{\nabla} \times \vec{B}) / \sigma$ is the Ohmic electric field. The conductivity $\sigma$ is large during most of the thermodynamic history of the Universe, since it is proportional to the temperature when the temperature is much larger than the mass of the corresponding species. The contribution of the Ohmic Poynting vector will therefore be neglected in comparison with the other components of the magnetic energy-momentum tensor. Introducing the density contrast on comoving orthogonal hypersurfaces, i.e.

$$
\epsilon_{\mathrm{m}}(\tau, \vec{x})=\frac{\delta \rho_{\mathrm{t}}+\delta \rho_{\mathrm{B}}}{\rho_{\mathrm{t}}}-3 \mathcal{H}\left(1+w_{\mathrm{t}}\right) V_{\mathrm{t}},
$$

the Hamiltonian constraint (2.5) takes the peculiar form

$$
\nabla^{2} \Psi=4 \pi G a^{2} \rho_{\mathrm{t}} \epsilon_{\mathrm{m}},
$$

which is (just formally) analog to the Poisson equation typical of the nonrelativistic treatment of gravitational inhomogeneities. Another relevant pair of gauge-invariant quantities is represented by

$$
\begin{gathered}
\zeta=-\Psi-\frac{\mathcal{H}\left(\delta \rho_{\mathrm{t}}+\delta \rho_{\mathrm{B}}\right)}{\rho_{\mathrm{t}}^{\prime}}, \\
\mathcal{R}=-\Psi-\frac{\mathcal{H}\left(\mathcal{H} \Phi+\Psi^{\prime}\right)}{4 \pi G a^{2} \rho_{\mathrm{t}}(w+1)} .
\end{gathered}
$$

The variable $\zeta[44,45]$ defined in Eq. (2.9), if evaluated in the gauge where the spatial curvature is uniform, is proportional to the total density contrast [as it can be directly checked by using Eq. (2.3)]. The variable $\mathcal{R}$ [45,46], defined in Eq. (2.10), if evaluated on comoving orthogonal hypersurfaces, coincides with the fluctuations of the spatial curvature. It is clear that the three gauge-invariant variables defined, respectively, in Eqs. (2.7), (2.9), and (2.10) are all related by the Hamiltonian constraint. Inserting Eq. (2.3) into Eq. (2.9) and taking into account Eqs. (2.5), (2.8), and (2.10) the Hamiltonian constraint can be expressed in one of the following two equivalent forms:

$$
\begin{gathered}
\zeta=\mathcal{R}+\frac{\nabla^{2} \Psi}{12 \pi G a^{2} \rho_{\mathrm{t}}\left(1+w_{\mathrm{t}}\right)}, \\
\zeta=\mathcal{R}+\frac{\epsilon_{\mathrm{m}}}{3\left(1+w_{\mathrm{t}}\right)},
\end{gathered}
$$

where Eq. (2.12) follows from Eq. (2.11) making use of the generalized Poisson Eq. (2.8). It is relevant to remark that when the wavelengths are larger than the Hubble radius, at a given epoch,

$$
\zeta \simeq \mathcal{R}+\mathcal{O}\left(\frac{k^{2}}{a^{2} H^{2}}\right), \quad \epsilon_{\mathrm{m}} \simeq \mathcal{O}\left(\frac{k^{2}}{a^{2} H^{2}}\right) .
$$

In spite of the physical differences between $\zeta, \mathcal{R}$, and $\epsilon_{\mathrm{m}}$ the explicit solution of the whole system in terms of one of these variables allows computation of the others. From the fluctuation of the covariant conservation equation of the total plasma the following equation can be easily obtained:

$\delta \rho_{\mathrm{t}}^{\prime}+3 \mathcal{H}\left(\delta \rho_{\mathrm{t}}+\delta p_{\mathrm{t}}\right)-3\left(p_{\mathrm{t}}+\rho_{\mathrm{t}}\right) \Psi^{\prime}+\left(p_{\mathrm{t}}+\rho_{\mathrm{t}}\right) \theta_{\mathrm{t}}=0$.

Using now Eq. (2.9) inside Eq. (2.14), a first-order differential equation for $\zeta$ emerges and it is given by

$$
\zeta^{\prime}=-\frac{\mathcal{H}}{\rho_{\mathrm{t}}\left(1+w_{\mathrm{t}}\right)} \delta p_{\mathrm{nad}}+\frac{\mathcal{H}\left(3 c_{\mathrm{st}}^{2}-1\right)}{3 \rho_{\mathrm{t}}\left(1+w_{\mathrm{t}}\right)} \delta \rho_{\mathrm{B}}-\frac{\theta_{\mathrm{t}}}{3} .
$$

The term $\delta p_{\text {nad }}$ in Eq. (2.15) accounts for the nonadiabatic pressure inhomogeneities; the following decomposition of the total pressure fluctuation

$$
\delta p_{\mathrm{t}}=\left(\frac{\delta p_{\mathrm{t}}}{\delta \rho_{\mathrm{t}}}\right)_{\mathrm{s}} \delta \rho_{\mathrm{t}}+\left(\frac{\delta p_{\mathrm{t}}}{\delta \mathrm{s}}\right)_{\rho_{\mathrm{t}}} \delta \mathrm{s} \equiv c_{\mathrm{st}}^{2} \delta \rho_{\mathrm{t}}+\delta p_{\mathrm{nad}}
$$

has been tacitly assumed in Eqs. (2.14) and (2.15). While the first (adiabatic) contribution gives the pressure fluctuation produced by the inhomogeneous density fluctuation when the specific entropy is constant (i.e. $\delta \varsigma=0$ ), the second (nonadiabatic) contribution arises even if the density is unperturbed (i.e. $\delta \rho_{\mathrm{t}}=0$ ) but the plasma possesses many different components (for instance, in the prerecom- 
binantion plasma, photons, baryons, neutrinos, and dark matter).

The evolution of $\mathcal{R}$ can be obtained, in similar terms, from the equations of $\Phi$ and $\Psi$, i.e.

$$
\begin{aligned}
\Psi^{\prime \prime} & +\mathcal{H}\left(\Phi^{\prime}+2 \Psi^{\prime}\right)+\left(2 \mathcal{H}^{\prime}+\mathcal{H}^{2}\right) \Phi+\frac{1}{3} \nabla^{2}(\Phi-\Psi) \\
& =4 \pi G a^{2}\left(\delta p_{\mathrm{t}}+\delta p_{\mathrm{B}}\right), \\
& \left(\partial_{i} \partial^{j}-\frac{1}{3} \delta_{i}^{j} \nabla^{2}\right)(\Phi-\Psi)=8 \pi G a^{2}\left(\Pi_{i}^{j}+\bar{\Pi}_{i}^{j}\right),
\end{aligned}
$$

where $\Pi_{i}^{j}$ is the anisotropic stress of the fluid and

$$
\bar{\Pi}_{i}^{j}=\frac{1}{4 \pi a^{4}}\left(B_{i} B^{j}-\frac{\delta_{i}^{j}}{3} B^{2}\right)
$$

is the anisotropic stress of the magnetic field. Inserting Eq. (2.10) into Eq. (2.17) and recalling Eq. (2.16) the evolution equation of $\mathcal{R}$ can be written as

$$
\begin{aligned}
\mathcal{R}^{\prime}= & -\frac{\mathcal{H}}{\rho_{\mathrm{t}}\left(1+w_{\mathrm{t}}\right)} \delta p_{\text {nad }}+\frac{\mathcal{H}\left(3 c_{\mathrm{st}}^{2}-1\right)}{3 \rho_{\mathrm{t}}\left(1+w_{\mathrm{t}}\right)} \delta \rho_{\mathrm{B}} \\
& +\frac{\mathcal{H}}{12 \pi G a^{2}\left(p_{\mathrm{t}}+\rho_{\mathrm{t}}\right)} \nabla^{2}(\Phi-\Psi) \\
& -\frac{\mathcal{H} c_{\mathrm{st}}^{2}}{4 \pi G a^{2}\left(p_{\mathrm{t}}+\rho_{\mathrm{t}}\right)} \nabla^{2} \Psi .
\end{aligned}
$$

By now subtracting Eq. (2.20) from Eq. (2.15) and by using Eqs. (2.12) and (2.13), the appropriate equation of $\epsilon_{\mathrm{m}}$ can be obtained after simple algebra:

$$
\epsilon_{\mathrm{m}}^{\prime}-3 \mathcal{H} w_{\mathrm{t}} \epsilon_{\mathrm{m}}=-\left(1+w_{\mathrm{t}}\right) \theta_{\mathrm{t}}-3 \mathcal{H}\left(1+w_{\mathrm{t}}\right) \Pi_{\mathrm{t}}
$$

where the notation

$$
\partial_{i} \partial^{j} \Pi_{j}^{i}+\partial_{i} \partial^{j} \bar{\Pi}_{i}^{j}=\left(p_{\mathrm{t}}+\rho_{\mathrm{t}}\right) \nabla^{2} \Pi_{\mathrm{t}}
$$

has been used. If there are collisionless particles in the plasma (like neutrinos, after weak interactions have fallen out of thermal equilibrium),

$$
\left(p_{\mathrm{t}}+\rho_{\mathrm{t}}\right) \nabla^{2} \Pi_{\mathrm{t}}=\left(p_{\nu}+\rho_{\nu}\right) \nabla^{2} \Pi_{\nu}+\left(p_{\gamma}+\rho_{\gamma}\right) \nabla^{2} \Pi_{\mathrm{B}} .
$$

Concerning Eq. (2.23) two comments are in order. I referred the magnetic anisotropic stress to the photon background. This is natural since, for typical length-scales larger than the magnetic diffusivity scale, both quantities redshift at the same rate with the expansion of the Universe. The second comment involves the relevance of the anisotropic stress. As discussed in relation with the estimate of the CMB autocorrelation induced by magnetic fields, the magnetic anisotropic stress is of upmost importance at intermediate scales. In particular the interplay with the neutrino anisotropic stress leads to the correct initial conditions for the magnetized CMB anisotropies.
However, in the present paper I will be mostly concerned with the largest scales and will try to assess what could be the influence, on those scales, of slightly different thermal histories of the Universe. For typical wavelengths larger than the Hubble radius at recombination the anisotropic stress can be neglected in the first approximation. If needed, however, it can be included with the standard iterative procedure $[47,48]$ (see also [49]) where, to lowest order, the solution is the one where $\Phi \simeq \Psi$.

\section{DIFFERENT THERMAL HISTORIES}

By looking at Eq. (2.15) it is clear that, to leading order in $k^{2} \tau^{2}$, the evolution of $\zeta$ (and of $\mathcal{R}$ ) is determined by three independent pieces of information:

(i) the presence (or absence) of nonadiabatic pressure fluctuations;

(ii) presence (or absence) of large-scale magnetic fields;

(iii) the specific time dependence of $w_{\mathrm{t}}$ and $c_{\mathrm{st}}^{2}$.

The simplest possible situation compatible with the presence of super-Hubble magnetic fields arises when the Universe becomes suddenly dominated by radiation at the end of inflation. This is, incidentally, also the underlying assumption in the standard $\Lambda$ CDM scenario. The Universe will then become dominated by dusty matter at a redshift ${ }^{5} z_{\text {eq }} \simeq 3200$. If inflation was driven by a single inflaton and if other spectator fields with scale-invariant spectra were absent, then it is also rather plausible to enforce the condition $\delta p_{\text {nad }}=0$. In this situation, the exact solution of Eqs. (2.1), (2.2), and (2.3) implies that

$$
w_{\mathrm{t}}(\alpha)=\frac{1}{3(\alpha+1)}, \quad c_{\mathrm{st}}^{2}(\alpha)=\frac{4}{3(3 \alpha+4)},
$$

where

$$
\begin{array}{rlrl}
\alpha(\tau)=\frac{a}{a_{\mathrm{eq}}}=x^{2}+2 x, & x=\frac{\tau}{\tau_{1}}, & \frac{a_{0}}{a_{\mathrm{eq}}}=\frac{h_{0}^{2} \Omega_{\mathrm{M} 0}}{h_{0}^{2} \Omega_{\mathrm{R} 0}} \\
\frac{a_{0}}{a_{\mathrm{eq}}}=\frac{h_{0}^{2} \Omega_{\mathrm{M} 0}}{h_{0}^{2} \Omega_{\mathrm{R} 0}}, & \tau_{\mathrm{eq}}=(\sqrt{2}-1) \tau_{\mathrm{eq}} .
\end{array}
$$

Using Eqs. (3.1) and (3.2) inside Eq. (2.15) and changing the variable from $\tau$ to $\alpha$ the following expression can be obtained:

$$
\begin{gathered}
\frac{d \zeta}{d \alpha}=-\frac{3 R_{\gamma} \Omega_{\mathrm{B}}}{(3 \alpha+4)^{2}}, \quad R_{\gamma}=\frac{\rho_{\gamma}(\tau)}{\rho_{\mathrm{R}}(\tau)}, \\
\Omega_{\mathrm{B}}(\tau, \vec{x})=\frac{\delta \rho_{\mathrm{B}}(\tau, \vec{x})}{\rho_{\gamma}(\tau)} .
\end{gathered}
$$

\footnotetext{
${ }^{5}$ When not otherwise stated it will be assumed that $h_{0}^{2} \Omega_{\mathrm{M} 0} \simeq$ 0.1326 , where $\Omega_{\mathrm{M} 0}$ denotes the present fraction in dusty matter. This value for the total critical fraction of matter emerges, in the context of the $\Lambda \mathrm{CDM}$ paradigm, when the WMAP data [22,23] are combined with all the remaining cosmological data sets of different origin (excluding weak lensing data).
} 
In Eq. (3.3) the magnetic energy density has been expressed in units of the photon energy density and $R_{\gamma}$ denotes the photon fraction in the post-inflationary radiation background. After neutrinos decouple for temperatures of the order of the $\mathrm{MeV}$ the photon fraction will be related with the neutrino fraction as $R_{\gamma}=1-R_{\nu}$ where $R_{\nu}=0.405$ (for three neutrino families). Direct integration of Eq. (3.3) gives the sought result, namely

$$
\zeta(k, \tau)=\zeta_{*}(k)-\frac{3 R_{\gamma} \Omega_{\mathrm{B}(k)} \alpha(\tau)}{4(3 \alpha(\tau)+4)} .
$$

The constant $\zeta_{*}(k)$ stands for the adiabatic mode produced (for instance during a phase of conventional inflation). After matter-radiation equality (but before recombination) to leading order in $k^{2} \tau^{2}$ I will have, from Eq. (2.11), that

$$
\begin{aligned}
\mathcal{R}\left(k, \tau_{\mathrm{rec}}\right) & \simeq \zeta_{*}(k)-\frac{R_{\gamma} \Omega_{\mathrm{B}}(k)}{4}\left(\frac{3 \alpha_{\mathrm{rec}}}{3 \alpha_{\mathrm{rec}}+4}\right)+\mathcal{O}\left(k^{2} \tau_{\mathrm{rec}}^{2}\right), \\
\alpha_{\mathrm{rec}} & \simeq \frac{z_{\mathrm{eq}}+1}{z_{\mathrm{rec}}+1}=3.07\left(\frac{h_{0}^{2} \Omega_{\mathrm{M} 0}}{0.134}\right) .
\end{aligned}
$$

This result can be easily interpreted from the physical point of view. When the rate of expansion increases from radiation to matter the barotropic index (and the sound speed) are both decreasing from $1 / 3$ to 0 . Thus the overall effect on the source term for the evolution of curvature perturbations implies that the magnetic contribution tends to cancel the contribution of the adiabatic mode.

In the context of this reference scenario when the Universe only passes, after inflation, from radiation to matter also $\epsilon_{\mathrm{m}}$ and $\theta_{\mathrm{t}}$ can be computed, respectively, from Eqs. (2.6) and (2.8). It suffices to extract $\Psi$ from $\zeta$ and then use the aforementioned constraints. Going to Fourier space the result of this manipulation will be

$$
\begin{gathered}
\epsilon_{\mathrm{m}}(k, \tau)=\frac{k^{2} \tau_{1}^{2}}{12 \alpha \sqrt{\alpha+1}}\left[\zeta_{*}(k) W_{1}(\alpha)\right. \\
\left.-\frac{3}{4} R_{\gamma} \Omega_{\mathrm{B}}(k) \mathcal{W}_{2}(\alpha)\right], \\
\theta_{\mathrm{t}}(k, \tau)=-\frac{k^{2} \tau_{1}}{2}\left\{\left[\frac{\alpha}{\sqrt{\alpha+1}}-\frac{\mathcal{W}_{1}(\alpha)}{2 \alpha^{2}}\right] \zeta_{*}(k)\right. \\
\left.+\frac{3}{8} R_{\gamma} \Omega_{\mathrm{B}}(k)\left[\frac{\mathcal{W}_{2}(\alpha)}{\alpha^{2}}-\frac{2 \alpha^{2}}{(3 \alpha+4) \sqrt{\alpha+1}}\right]\right\},
\end{gathered}
$$

where

$$
\begin{aligned}
& \mathcal{W}_{1}(\alpha)=\frac{2}{15 \sqrt{\alpha+1}}\{16[\sqrt{\alpha}-1]+\alpha[\alpha(9 \alpha+2)-8]\}, \\
& \mathcal{W}_{2}(\alpha)=\frac{2}{5 \sqrt{\alpha+1}}\{16[1-\sqrt{\alpha+1}]+\alpha[8+\alpha(\alpha-2)]\} .
\end{aligned}
$$

Thanks to the success of big-bang nucleosynthesis, it is rather plausible to imagine that the Universe was already dominated by radiation for temperatures as large as few $\mathrm{MeV}$. However, prior to that time there are no direct probes of the expansion rate of the Universe. In a somehow indirect way this could be achieved through the study of the cosmic background of gravitational waves. At the moment, for instance, there is no compelling evidence of the fact that, after inflation, the Universe became suddenly dominated by radiation. It is, on the contrary, rather reasonable that, before settling on a radiation-dominated stage of expansion the Universe passed through a phase where, for instance, the rate of expansion was smaller than the Hubble rate during radiation. This occurrence is realized when the total barotropic index of the sources driving the geometry satisfies $1 / 3<w_{\mathrm{t}} \leq 1$. In this case the sources are said to be stiffer than radiation [50]. The stiffest equation of state I can imagine is the one where the barotropic index is 1 which implies that the sound speed coincides with the speed of light. The case of speed of sound equal to speed of light is the one contemplated by the so-called Zeldovich model (see [51] and references therein). A similar kind of evolution arises in quintessential inflationary models where the inflaton and the quintessence field are unified in a single degree of freedom. In this case (as in the more general case of stiffer phases [50]) the spectrum of relic gravitons presents a sharp increase [52] and a peak [53] which are potentially accessible to direct observations. In the context of quintessential inflation the stiff epoch is effectively dominated by the kinetic energy of the inflaton. Stiff phases also arise in brane-world scenarios [54] where the calculation of the graviton spectrum mirrors the fourdimensional case [52,53].

The transition from a stiff epoch to a radiationdominated phase of expansion can be understood in terms of the following exact solution:

$$
\alpha=\frac{a}{a_{\mathrm{r}}}=\sqrt{y^{2}+2 y}, \quad y=\frac{\tau}{\tau_{1}}, \quad \tau_{1}=\frac{2}{\lambda H_{*}},
$$

where

$$
a_{\mathrm{r}}=a_{*} \sqrt{\frac{\rho_{\mathrm{S}^{*}}}{\rho_{\mathrm{R} *}}} \simeq \frac{M_{\mathrm{P}}}{H_{*}}, \quad \lambda=a_{*} \frac{H_{*}}{M_{\mathrm{P}}} .
$$

Eqs. (3.9) and (3.10) are solutions of Eqs. (2.1), (2.2), and (2.3) when the sources of the geometry are given in terms of a mixture of radiation and of a stiff fluid with $p_{\mathrm{s}}=\rho_{\mathrm{s}}$. Eqs. (3.9) and (3.10) parametrize the physical situation where, at $\tau_{*}$ some (small) amount of radiation is present in comparison with the stiff contribution (i.e. $\rho_{\mathrm{R} *} \ll \rho_{\mathrm{S} *}$ ). In the context of quintessential inflationary models [55] the initial amount of radiation comes indeed from quantum fluctuations [56] and, consequently, $\rho_{\mathrm{R} *} \simeq H_{*}^{4}$. This occurrence implies, in turn, the hierarchy between the initial scale factor at $\tau_{*}$ and the scale factor at the onset of 
radiation (i.e. $a_{\mathrm{r}}$ is determined by $M_{\mathrm{P}} / H_{*}$ ). Since $H_{*}<$ $10^{-6} M_{\mathrm{P}}$, the duration of the stiff phase is correctly bounded because $H_{\mathrm{r}}>H_{\mathrm{bbn}}$. According to Eq. (2.4), the barotropic index and the sound speed are

$$
w_{\mathrm{t}}=\frac{1}{3} \frac{\alpha^{2}+3}{\alpha^{2}+1}, \quad c_{\mathrm{st}}^{2}=\frac{2 \alpha^{2}+9}{3\left(2 \alpha^{2}+3\right)} .
$$

Using therefore Eq. (3.11) inside Eq. (2.15) and changing variable from $\tau$ to $\alpha$ :

$$
\frac{d \zeta}{d \alpha}=\frac{3 R_{\gamma} \Omega_{\mathrm{B}} \alpha}{\left(2 \alpha^{2}+3\right)^{2}} .
$$

Integrating Eq. (3.12) between $\alpha=0$ and $\alpha_{\mathrm{f}}$ gives

$$
\zeta(k, \tau)=\zeta_{*}(k)+\frac{R_{\gamma} \Omega_{\mathrm{B}(k)} \alpha_{\mathrm{f}}^{2}}{2\left(2 \alpha_{\mathrm{f}}^{2}+3\right)},
$$

where $\alpha_{\mathrm{f}} \gg 1$ and it is defined deep in the radiation epoch. This result implies that the magnetic contribution enhances (rather than canceling) the adiabatic term $\zeta_{*}(k)$ of inflationary origin.

As in the case of the radiation-matter transition the total density contrast and the total peculiar velocity can be computed. In Fourier space the result is

$$
\begin{aligned}
\epsilon_{\mathrm{m}}(k, \tau)= & -\frac{k^{2} \tau_{*}^{2}}{6 \sqrt{\alpha^{2}+1}}\left[2 \zeta_{*}(k) \mathcal{W}_{3}(\alpha)\right. \\
& \left.+R_{\gamma} \Omega_{\mathrm{B}}(k) \mathcal{W}_{4}(\alpha)\right], \\
\theta_{\mathrm{t}}(k, \tau)= & -k^{2} \tau_{*}\left\{\left[\frac{\alpha^{2}}{\sqrt{\alpha^{2}+1}}-\frac{2 \mathcal{W}_{3}(\alpha)}{\alpha^{2}}\right] \zeta_{*}(k)\right. \\
+ & \frac{R_{\gamma} \Omega_{\mathrm{B}}(k)}{2}\left[\frac{\alpha^{4}}{\left(2 \alpha^{2}+3\right) \sqrt{\alpha^{2}+1}}\right. \\
& \left.\left.-\frac{2}{\alpha^{2}} W_{4}(\alpha)\right]\right\},
\end{aligned}
$$

where

$$
\begin{aligned}
& \mathcal{W}_{3}(\alpha)=\frac{4}{3}\left[\left(\alpha^{2}+1\right)^{3 / 2}-1\right]-\frac{2 \alpha^{2}}{\sqrt{\alpha^{2}+1}}, \\
& \mathcal{W}_{3}(\alpha)=\frac{2}{3}\left[\left(\alpha^{2}+1\right)^{3 / 2}+8\right]-\frac{2\left(2 \alpha^{2}+3\right)}{\sqrt{\alpha^{2}+1}} .
\end{aligned}
$$

This result can be generalized when the stiff phase is parametrized in terms of a source $p_{\mathrm{s}}=\delta \rho_{\mathrm{s}}$ with $1<\delta<$ $1 / 3$. Repeating the same steps outlined above I will have that the dependence of the barotropic index and of the sound speed upon the scale factor are given, in this case, by

$$
w_{\mathrm{t}}=\frac{1}{3}\left(\frac{3 \delta+\alpha^{3 \delta-1}}{1+\alpha^{3 \delta-1}}\right), \quad c_{\mathrm{st}}^{2}=\frac{9 \delta(\delta+1)+4 \alpha^{3 \delta-1}}{3\left[3(\delta+1)+4 \alpha^{3 \delta-1}\right]} .
$$

The equation for $\zeta$ and its related solution can then be written as

$$
\begin{gathered}
\frac{d \zeta}{d \alpha}=3 R_{\gamma} \Omega_{\mathrm{B}} \frac{(\delta+1)(3 \delta-1) \alpha^{3 \delta-2}}{\left[3(\delta+1)+4 \alpha^{3 \delta-1}\right]^{2}}, \\
\zeta(k, \tau)=\zeta_{*}(k)+R_{\gamma} \Omega_{\mathrm{B}}(k) \frac{\alpha_{\mathrm{f}}^{3 \delta-1}}{4 \alpha_{\mathrm{f}}^{3 \delta-1}+3(\delta+1)} .
\end{gathered}
$$

As in the other two cases $\epsilon_{\mathrm{m}}$ and $\theta_{\mathrm{t}}$ can be easily computed:

$$
\begin{aligned}
\epsilon_{\mathrm{m}}(k, \tau)= & \frac{k^{2} \tau_{*}^{2}}{9 \delta^{2}-1} \frac{\alpha^{3(\delta-1) / 2}}{\sqrt{1+\alpha^{3 \delta-1}}}\left[\mathcal{W}_{5}(\alpha, \delta) \zeta_{*}(\vec{k})\right. \\
& \left.+R_{\gamma} \mathcal{W}_{6}(\alpha, \delta) \Omega_{\mathrm{B}}(k)\right],
\end{aligned}
$$

$$
\begin{aligned}
\theta_{\mathrm{t}}(k, \tau)= & \frac{k^{2} \tau_{*}}{\sqrt{3 \delta+1}} \frac{\alpha^{(3 \delta+1) / 2}}{\sqrt{\alpha^{3 \delta-1}+1}} \\
& \times\left\{\zeta_{*}(k)\left[1-\frac{\sqrt{\alpha^{3 \delta-1}+1}}{2(3 \delta-1)} \frac{W_{5}(\alpha, \delta)}{\alpha^{(3 \delta+5) / 2}}\right] R_{\gamma} \Omega_{\mathrm{B}}(\vec{k})\right. \\
& \times\left[\frac{\alpha^{3 \delta-1}}{4 \alpha^{3 \delta-1}+3(\delta+1)}\right. \\
& \left.\left.-\frac{\sqrt{\alpha^{3 \delta-1}+1}}{\alpha^{(3 \delta+5) / 2}} \frac{W_{6}(\alpha, \delta)}{2(3 \delta-1)}\right]\right\}
\end{aligned}
$$

where

$$
\begin{aligned}
& W_{5}(\alpha, \delta)=\int_{0}^{s(\alpha)} \frac{4 s+3(\delta+1)}{(s+1)^{3 / 2}} s^{(7-3 \delta) / 2(3 \delta-1)} d s, \\
& \mathcal{W}_{6}(\alpha, \delta)=\int_{0}^{s(\alpha)} \frac{s^{(5+3 \delta) / 2(3 \delta-1)}}{(s+1)^{3 / 2}} d s .
\end{aligned}
$$

The upper limit of integration in Eqs. (3.22) corresponds to $s(\alpha)=\alpha^{3 \delta-1}$. For each value of $\delta$ the above integrals can be evaluated in explicit terms.

Suppose now the investigation of a three stage model where the Universe expanded, initially, at a rate that was slower than radiation then got trapped in a radiation phase which turned subsequently into an epoch dominated by dusty matter. In this case the barotropic index and the sound speed are given, respectively, by

$$
\begin{array}{r}
w_{\mathrm{t}}=\frac{1}{3} \frac{3+\alpha^{2}}{1+\alpha^{2}+b \alpha^{3}}, \quad b=\frac{\rho_{\mathrm{M} *}}{\rho_{\mathrm{s} *}}\left(\frac{\rho}{\rho^{2}}\right. \\
c_{\mathrm{st}}^{2}=\frac{18+4 \alpha^{2}}{18+12 \alpha^{2}+9 b \alpha^{3}} .
\end{array}
$$

In this case the evolution equation of curvature perturbations is given by

$$
\frac{d \zeta}{d \alpha}=-3 R_{\gamma} \Omega_{\mathrm{B}} \frac{\alpha\left(\alpha^{3} b-4\right)}{\left(3 \alpha^{3} b+4 \alpha^{2}+6\right)^{2}} .
$$

The (approximate) solution of Eq. (3.25) in the matterdominated epoch reads 


$$
\zeta(k, \tau) \simeq \zeta_{*}(k)+\frac{R_{\gamma} \Omega_{\mathrm{B}}(k)}{3 \alpha_{\mathrm{rec}}+4} .
$$

The result of Eq. (3.26) shows an extra suppression of a factor $\alpha_{\text {rec }} \simeq 1 / 3$ in comparison with Eq. (3.5). This suppression can be easily understood. The evolution described by Eqs. (3.23) and (3.24) implies that, right after inflation, the Universe expands slower than radiation. When radiation kicks in, the curvature perturbations are enhanced. Later on, when dusty matter becomes dominant (around matter-radiation equality) the magnetized contribution tends, again, to cancel the preexisting adiabatic mode. The net result of the initial increase and of the subsequent decrease of $\zeta$ is given in Eq. (3.26) and it is a bit different from the result of Eq. (3.5) where the intermediate stiff phase was absent. The following conclusions can then be drawn:

(i) if the Universe is dominated by radiation from the end of inflation (as it happens in the case of the standard evolution) the magnetized contribution and the adiabatic mode have opposite sign;

(ii) if, prior to the electroweak epoch, the thermodynamic history of the Universe deviates from a radiation epoch, the magnetized contribution is more suppressed in comparison with the standard case.

In the framework of conventional inflationary models this type of deviation from the standard scenario is pretty general. By general I mean that, within the current bounds on the expansion of the Universe arising from big-bang nucleosynthesis I can just imagine drastic deviation from the radiation-dominated evolution between the end of inflation and, say, neutrino decoupling. In fact, deviation from a radiation-dominated evolution after (or right before) big-bang nucleosynthesis will necessarily jeopardize the production of the abundances of the light elements.

Up to now it has been assumed that the fluids of the primeval plasma do not exchange energy. Let me now address this interesting situation that could arise, for instance, in the course of reheating. Therefore imagine that a matter fluid (which can model the coherent oscillations of the inflaton) decays into massless particles. This dynamics can be parametrized by the following system ${ }^{6}$ :

$$
\begin{gathered}
\dot{H}=-4 \pi G\left(\frac{4}{3} \rho_{\mathrm{r}}+\rho_{\mathrm{m}}\right), \\
\dot{\rho}_{\mathrm{m}}=-3 H \rho_{\mathrm{m}}-\Gamma \rho_{\mathrm{m}}, \\
\dot{\rho}_{\mathrm{r}}=-4 H \rho_{\mathrm{r}}+\Gamma \rho_{\mathrm{m}} .
\end{gathered}
$$

The Universe, initially dominated (right after inflation) by dusty matter, becomes rapidly dominated by radiation at a

\footnotetext{
${ }^{6}$ Here I passed from the conformal to the cosmic time parametrization. See the comments after Eqs. (2.1), (2.2), and (2.3).
}

rate that is controlled by $\bar{\Gamma}$. It should be immediately noticed that, by summing up Eqs. (3.28) and (3.29) the total energy density $\rho_{\mathrm{t}}=\rho_{\mathrm{r}}+\rho_{\mathrm{m}}$ is covariantly conserved, i.e. $\dot{\rho}_{\mathrm{t}}+3 H\left(\rho_{\mathrm{t}}+p_{\mathrm{t}}\right)=0$. Eqs. (3.28) and (3.29) can be approximately solved:

$$
\rho_{\mathrm{m}}(t)=\rho_{\mathrm{r}}\left(t_{\mathrm{i}}\right)\left(\frac{a_{\mathrm{i}}}{a}\right)^{3} e^{-\bar{\Gamma}\left(t-t_{\mathrm{i}}\right)}, \quad \rho_{\mathrm{r}}(t) \simeq \rho_{\mathrm{r}}\left(t_{\mathrm{d}}\right)\left(\frac{a_{\mathrm{d}}}{a}\right)^{4},
$$

where $t_{\mathrm{d}} \simeq \bar{\Gamma}^{-1}$. As a consequence of the relations of Eq. (3.30) one can also say that

$$
\frac{\rho_{\mathrm{m}}}{\rho_{\mathrm{r}}} \simeq \frac{H_{\mathrm{i}}^{2}}{H_{\mathrm{d}}^{2}}\left(\frac{a_{\mathrm{i}}}{a}\right)^{3}\left(\frac{a}{a_{\mathrm{d}}}\right)^{4} e^{-\bar{\Gamma}\left(t-t_{\mathrm{i}}\right)} \simeq(t \bar{\Gamma})^{1 / 2} e^{-\bar{\Gamma}\left(t-t_{\mathrm{i}}\right)} .
$$

In this situation the total barotropic index and the total sound speed are slightly modified thanks to the physical differences of the system. In particular

$$
c_{\mathrm{st}}^{2}=\frac{4}{3} \frac{\rho_{\mathrm{r}}}{4 \rho_{\mathrm{r}}+3 \rho_{\mathrm{m}}}-\frac{\bar{\Gamma}}{3 H} \frac{\rho_{\mathrm{m}}}{4 \rho_{\mathrm{r}}+3 \rho_{\mathrm{m}}} .
$$

From Eq. (3.32) is clear that $c_{\mathrm{st}}^{2} \rightarrow 1 / 3$ when $\bar{\Gamma} \gg H$.

The evolution equations of curvature perturbations to be solved read

$$
\begin{gathered}
\dot{\zeta}=-\frac{H}{p_{\mathrm{t}}+\rho_{\mathrm{t}}} \delta p_{\mathrm{nad}}+\frac{H}{p_{\mathrm{t}}+\rho_{\mathrm{t}}}\left(c_{\mathrm{st}}^{2}-\frac{1}{3}\right) \delta \rho_{\mathrm{B}}, \\
\dot{\zeta}_{\mathrm{m}}=\frac{\dot{g}}{g} \zeta_{\mathrm{m}}-\bar{\Gamma} g \frac{\dot{H}}{H^{2}} \zeta,
\end{gathered}
$$

where $g=H /(\bar{\Gamma}+3 H)$. Let me now concentrate, as discussed above, on the conventional situation where the nonadiabatic modes are totally absent. In this case, initially, $\delta p_{\text {nad }} \propto\left(\zeta_{\mathrm{r}}-\zeta_{\mathrm{m}}\right) \simeq 0$ and $\zeta_{\mathrm{r}}=\zeta_{\mathrm{m}} \simeq \zeta_{*}$. Using Eq. (3.32) inside Eq. (3.33) I will simply have that

$$
\dot{\zeta}=-\frac{H \delta \rho_{\mathrm{B}} \rho_{\mathrm{m}}}{g\left(4 \rho_{\mathrm{r}}+3 \rho_{\mathrm{m}}\right)^{2}} .
$$

Recalling that, after $t_{\mathrm{d}}, \bar{\Gamma} \gg H$ and $\rho_{\mathrm{m}}$ is exponentially suppressed, Eq. (3.35) can be written as

$$
\frac{d \zeta}{d x}=-\frac{R_{\gamma} \Omega_{\mathrm{B}}}{16} \sqrt{x} e^{-x}, \quad x=\bar{\Gamma} t .
$$

Integrating once Eq. (3.36) the result will be

$$
\zeta(k, y)=\zeta_{*}(k)-\frac{R_{\gamma} \Omega_{\mathrm{B}}(k)}{16} \int_{1}^{y} \sqrt{x} e^{-x} d x .
$$

The integral mentioned above can be done introducing the error function, i.e.

$$
\int_{1}^{y} \sqrt{x} e^{-x} d x=\frac{1}{e}-\frac{\sqrt{y}}{e^{y}}-\frac{\sqrt{\pi} \operatorname{Erf}(1)}{2}+\frac{\sqrt{\pi} \operatorname{Erf}(\sqrt{y})}{2} .
$$

Taking the limit $y \rightarrow \infty$, I then have 


$$
\zeta(k, t) \rightarrow \zeta_{*}(k)-\frac{R_{\gamma} \Omega_{\mathrm{B}}(k)}{16}\left(\frac{2+e \sqrt{\pi}-e \sqrt{\pi} \operatorname{Erf}(1)}{2 e}\right),
$$

that is, numerically,

$$
\zeta(k, t) \sim \zeta_{*}(k)-0.079 R_{\gamma} \Omega_{\mathrm{B}}(k) \sim \zeta_{*}(k)-\frac{R_{\gamma} \Omega_{\mathrm{B}}(k)}{12} .
$$

\section{BOUNDS ON DIFFERENT THERMAL HISTORIES}

The two-point correlation function of curvature perturbations will receive contributions, in general, from the adiabatic mode, from one (or more) nonadiabatic modes, and from the magnetic field. As argued in the previous sections, the simplest situation is the one where only the adiabatic mode is present together with the magnetized contribution that ought to be constrained. This choice is motivated by the experimental evidence that the Doppler peak structure of the temperature autocorrelations strongly suggests that, after equality, the large-scale curvature perturbations were predominantly adiabatic [22-26].

Consider therefore, the curvature perturbations present at recombination in the framework of the different thermal histories discussed in the previous section. The curvature perturbations can be written, in Fourier space, as ${ }^{7}$

$$
\zeta_{h}\left(k, \alpha_{\text {rec }}\right)=\zeta_{*}(k)+T_{h}\left(\alpha_{\text {rec }}, h_{0}^{2} \Omega_{\mathrm{M} 0}\right) \Omega_{\mathrm{B}}(k)
$$

where the subscript $h$ refers to each different thermal history and where $\zeta_{*}(k)$ represents the adiabatic contribution normalized to the large-scale value of the (ordinary) Sachs-Wolfe contribution to the temperature autocorrelations. The total (scalar) power spectrum $\mathcal{P}_{h}(k)$, i.e. the Fourier transform of the two-point function computed at different spatial locations but at the same time is defined as

$$
\left\langle\zeta_{h}(\tau, \vec{x}) \zeta_{h}(\tau, \vec{y})\right\rangle=\int d \ln k \mathcal{P}_{h}(k) \frac{\sin k r}{k r}, \quad r=|\vec{x}-\vec{y}| .
$$

From Eq. (4.1) and within the conventions summarized by Eq. (4.2),

$$
\left\langle\zeta_{h}(\vec{k}, \tau) \zeta_{h}(\vec{p}, \tau)\right\rangle=\frac{2 \pi^{2}}{k^{2}} \mathcal{P}_{h}(k) \delta^{(3)}(\vec{k}+\vec{p})
$$

where

$$
\begin{aligned}
\mathcal{P}_{h}(k)= & \mathcal{P}_{\zeta}(k)+T_{h}^{2}\left(\alpha_{\text {rec }}, h_{0}^{2} \Omega_{\mathrm{M} 0}\right) \mathcal{P}_{\Omega}(k) \\
& +2 T_{h}\left(\alpha_{\text {rec }}, h_{0}^{2} \Omega_{\mathrm{M} 0}\right) \sqrt{\mathcal{P}_{\zeta}(k)} \sqrt{\mathcal{P}_{\Omega}(k)} \cos \gamma,
\end{aligned}
$$

\footnotetext{
${ }^{7}$ The quantity $T_{h}\left(\alpha_{\mathrm{rec}}, h_{0}^{2} \Omega_{\mathrm{M} 0}\right)$ is the generalized transfer function that may change depending upon the specific thermal history labeled by the subscript $h$.
}

where $\gamma$ is the correlation angle between the adiabatic mode and the magnetized contribution. In Eq. (4.4) $\mathcal{P}_{\zeta}$ is the power spectrum of the adiabatic contribution defined as

$$
\begin{aligned}
& \mathcal{P}_{\zeta}=\mathcal{A}_{\zeta}\left(\frac{k}{k_{\mathrm{p}}}\right)^{n_{\zeta}-1}, \\
& \mathcal{A}_{\zeta}=\frac{2 \times 10^{4}}{9 T_{\mathrm{cmb}}^{2}} A\left(k_{\mathrm{p}}\right)=2.95 \times 10^{-9} A\left(k_{\mathrm{p}}\right) .
\end{aligned}
$$

In Eq. (4.5) $n_{\zeta}$ is the spectral index of the adiabatic mode and $\mathcal{A}_{\zeta}$ is the amplitude referred to the pivot scale $k_{\mathrm{p}}$. Following the WMAP conventions the value of the pivot scale will be fixed as $k_{\mathrm{p}}=0.002 \mathrm{Mpc}^{-1}$. The numerical factor appearing in the expression of $\mathcal{A}_{\zeta}$ has been obtained by taking consistently $T_{\mathrm{cmb}}=2.725 \times 10^{6}$ (expressed in units of $\mu K$ ). In the absence of any other contributions the WMAP 3-year data imply ${ }^{8} n_{\zeta} \simeq 0.947$ and $A\left(k_{\mathrm{p}}\right) \simeq 0.815$ when combined with the remaining cosmological data sets, i.e. supernovae and large-scale structure. ${ }^{9}$ In Eq. (4.5) the magnetic part of the correlation function is expressed as $[13,14]$

$$
\begin{aligned}
\mathcal{P}_{\Omega}(k) & =\mathcal{F}(\epsilon) \bar{\Omega}_{\mathrm{BL}}^{2}\left(\frac{k}{k_{\mathrm{L}}}\right)^{2 \epsilon}, \\
\mathcal{F}(\epsilon) & =\frac{4(6-\epsilon)(2 \pi)^{2 \epsilon}}{\epsilon(3-2 \epsilon) \Gamma^{2}(\epsilon / 2)},
\end{aligned}
$$

where $2 \epsilon$ is the spectral index of the magnetic energy density fluctuations and $k_{\mathrm{L}}$ is the magnetic pivot scale that will be defined in a moment. In Eq. (4.6)

$$
\bar{\Omega}_{\mathrm{BL}}=\frac{B_{\mathrm{L}}^{2}}{8 \pi \rho_{\gamma}}=7.56 \times 10^{-9}\left(\frac{B_{\mathrm{L}}}{\mathrm{nG}}\right)^{2} .
$$

In Eq. (4.7) $B_{\mathrm{L}}$ is the value of the magnetic field smoothed, through a Gaussian window function, over a typical comoving length $L$ which is related to the magnetic pivot scale as $L=2 \pi / k_{\mathrm{L}}$. In what follows the fiducial value $k_{\mathrm{L}}=\mathrm{Mpc}^{-1}$ will be consistently adopted. It is relevant to appreciate that $B_{\mathrm{L}}$ represents the smoothed magnetic field redshifted to the present epoch. This convention is a bit confusing but I will follow it since it is a common practice in the field. The source of the possible confusion is, in short, the following. The field $B_{\mathrm{L}}$ is the value of the magnetic field at recombination redshifted to the present epoch and assuming magnetic flux conservation which is well justified since the value of the conductivity is always

\footnotetext{
${ }^{8}$ In this analysis the values of the cosmological parameters will be fixed to the best fit values of the WMAP data combined with the other sets of cosmological observables. This choice is not crucial since the values of the cosmological parameters are purely illustrative. The essential features of the present analysis are unchanged if the best fit values of the WMAP data alone (or partially combined with the other data sets) are consistently assumed.

${ }^{9}$ The data $[32,35,36]$ are not included in the joined analysis.
} 
rather large. However, $\mathrm{B}_{\mathrm{L}}$ is not the present value of the magnetic field intensity observed in galaxies and galaxy clusters. The rationale for this statement is that during the gravitational collapse of protogalaxies the magnetic field intensity $B_{\mathrm{L}}$ will be amplified by compressional amplification and, probably, also by some dynamo action. Assuming just compressional amplification (which is the most certain aspect of the dynamics) the amplification factor may be of the order $10^{4}$ or even $10^{5}$.

Notice that, from Eq. (4.6) the nearly scale-invariant limit is achieved for $\epsilon<1$. Furthermore, as discussed elsewhere, the power spectrum of the magnetic energy density, being quartic in the field intensities, is computed in terms of the appropriate convolutions that have been evaluated, to get to Eq. (4.6), for $\epsilon<1$. It should be borne in mind that, in the opposite case (i.e. $\epsilon>1$ ) the magnetic energy density has a violet spectrum. This implies that, at very large length-scales the gravitational effect will be negligible and that, furthermore, the most significant bounds on the intensity of the magnetic field will come from comoving momenta close to the diffusion scale. Notice that the nearly scale-invariant spectrum is rather suggestive also because it would imply a nearly scaleinvariant spectrum of large-scale magnetic fields from galaxies, to clusters, to superclusters. This observation is, today, beyond our observational capabilities. However, by looking at recent determinations of magnetic fields in clusters and in some typical supercluster, it is indeed tempting to speculate that the resulting power spectrum of the magnetic energy density is nearly scale invariant.

The correlation function of curvature perturbations enters directly the determination of the ordinary Sachs-Wolfe contribution which is the dominant source of temperature anisotropies at large angular scales. Let me require that the magnetized contribution is always much smaller than the adiabatic contribution. So, I will assume, in a rather conservative approach, that the magnetized contribution is always smaller (by a factor $\eta$ ) than the adiabatic contribution. The parameter $\eta$ can be $\mathcal{O}\left(10^{-3}\right)$, in a conservative approach. Following this strategy the magnetic field intensity can be bounded in each thermal history of the Universe. Consider, for instance, the plots appearing in Fig. 1 where, on the vertical axis, the base 10 logarithm of the smoothed magnetic field is reported in units of $\mathrm{nG}$. In the plot at the left the three different lines correspond to the cases of the three different histories discussed in the previous sections. These three different histories will imply three different functions $T_{h}$ with $h=1,2,3$ and with, in general $T_{1} \neq T_{2} \neq T_{3}$. At late times the functions $T_{h}$ can be approximated as

$$
\begin{gathered}
T_{1}\left(\alpha_{\mathrm{rec}}, h_{0}^{2} \Omega_{\mathrm{M} 0}\right) \simeq \frac{3 R_{\gamma}}{4} \frac{\alpha_{\mathrm{rec}}}{3 \alpha_{\mathrm{rec}}+4}, \\
T_{2}\left(\alpha_{\mathrm{rec}}, h_{0}^{2} \Omega_{\mathrm{M} 0}\right) \simeq \frac{R_{\gamma}}{3 \alpha_{\mathrm{rec}}+4}, \\
T_{3}\left(\alpha_{\mathrm{rec}}, h_{0}^{2} \Omega_{\mathrm{M} 0}\right) \simeq \frac{R_{\gamma}}{12}\left(\frac{12 \alpha_{\mathrm{rec}}+4}{3 \alpha_{\mathrm{rec}}+4}\right) .
\end{gathered}
$$

The function $T_{1}$ arises in the simplest case, i.e. when the Universe, after a phase of conventional inflation, passes from a radiation-dominated epoch to the matter stage. In $T_{1}$ the reheating is assumed to be a sudden process. The function $T_{2}$ describes the situation when there is an intermediate stiff phase expanding at a rate slower than radiation. Finally the function $T_{3}$ arises when there is a prolonged reheating and then the Universe is, subsequently, dominated by radiation and matter. In Fig. 1 (plot at the left) I illustrate the bounds as a function of the magnetic spectral index. The region below each of the curves implies that the contribution of the magnetic field to the two-point function is smaller than $10^{-3}$.

The less restrictive case is, surprisingly enough, $T_{2}$. The rationale for this occurrence can be simply understood in terms of the results reported in the previous section. In the transition from the stiff phase to the radiation phase the magnetized component gets reduced while in the subse-

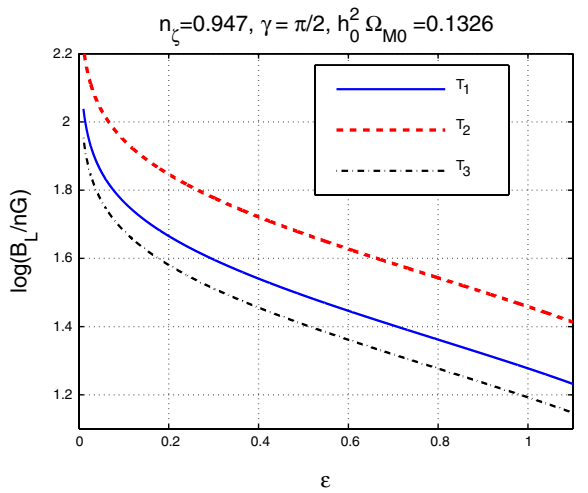

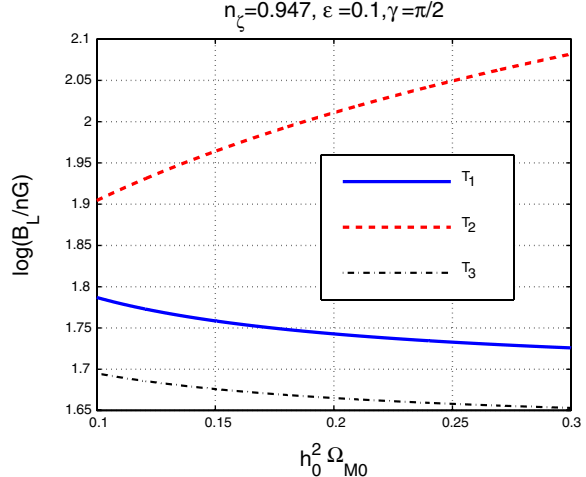

FIG. 1 (color online). The bounds on the smoothed magnetic field intensity are illustrated as a function of the magnetic spectral index (plot at the left) and as a function of the critical fraction in dusty matter (plot at the right). The pivot scales $k_{\mathrm{p}}$ and $k_{\mathrm{L}}$ are fixed, respectively, to $0.002 \mathrm{Mpc}^{-1}$ and $\mathrm{Mpc}^{-1}$. 

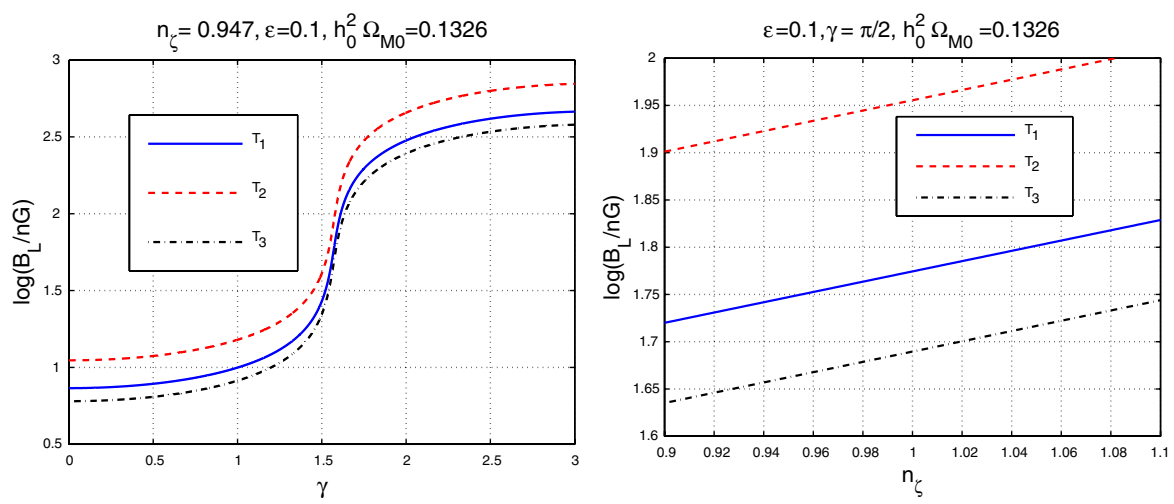

FIG. 2 (color online). The bounds on the smoothed magnetic field intensity are illustrated as a function of the correlation angle $\gamma$ (plot at the left) and as a function of the adiabatic spectral index $n_{\zeta}$ (plot at the right). All the other parameters are kept fixed to their fiducial values.

quent transition from radiation to matter the magnetized contribution gets enhanced. Since the two effects tend to cancel, the net result will be an overall suppression of the magnetized contribution. This dynamical suppression allows the magnetic field amplitude to be larger than in the other two cases (i.e. $T_{1}$ and $T_{3}$ ) where there is no "destructive" interference between the two subsequent effects.

In the plot at the right of Fig. 1 this pattern is confirmed with an extra piece of information: if the matter fraction increases the bound become loosers in the case of $T_{3}$ and a bit tighter in the case of $T_{1}$ and $T_{2}$. In fact, in Fig. 1 (plot at the right) the magnetic spectral index has been kept fixed while the matter fraction is allowed to move from the fiducial value $h_{0}^{2} \Omega_{\mathrm{M} 0}=0.1326$ which is the one assumed in the left plot of Fig. 1. The increase (or decrease) of the critical fraction of dusty matter simply means, physically, that the recombination epoch is either delayed or anticipated.

It should also be remarked that the case of prolonged reheating is the most constraining. This occurrence can be also simply understood from the considerations of the

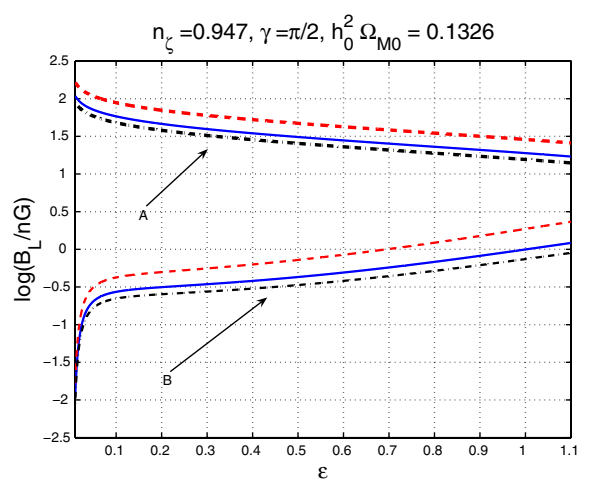

previous section. In the case of prolonged reheating $c_{\mathrm{st}}^{2} \neq$ $1 / 3$. So during this phase the curvature perturbations decrease and this decrease will interfere constructively with the usual radiation-matter transition since both contributions have the same sign. In Fig. 1 the value of the correlation angle has been fixed to $\pi / 2$ implying that the adiabatic and the magnetized modes are uncorrelated. Again I may relax this assumption and allow $\gamma$ to vary while all the other parameters are fixed to their fiducial values. This has been done in Fig. 2. As expected the bound is tighter in the case when the two components are correlated and looser when the two components are anticorrelated. In Fig. 2 (plot at the right) the variation of the adiabatic spectral index is illustrated always in the case where the two components are uncorrelated. It is now relevant to remark that while $P_{\zeta}(k)$ slightly decreases as $k$ increases, the opposite is true for $P_{\Omega}(k)$. It is therefore clear that, given this situation, the most constraining wave numbers are the largest, i.e. the small-scale behavior of the spectrum is the one that leads to the most stringent bounds. This consideration has been taken into account in deriving

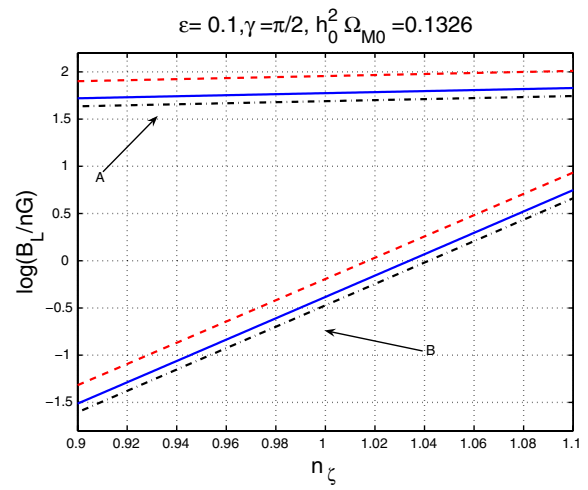

FIG. 3 (color online). The potential differences in the estimate of the diffusion scale are illustrated. In the plot at the left, the bunch of curves labeled by B corresponds to the estimate of the diffusion scale given in Eq. (4.12) while the curves labeled by A are the same illustrated in Fig. 1 (plot at the left). In the plot at the right the two classes of curves have illustrated the same phenomenon but in terms of the dependence on $n_{\zeta}$ and should be compared with the right plot appearing in Fig. 2. 
the bounds expressed by Figs. 1 and 2. In particular, it has been assumed that the diffusion scale is the one roughly associated with the Silk wave number, i.e.

$$
\frac{1}{k_{\mathrm{D}} \tau_{\text {rec }}}=9.63 \times 10^{-3}\left(\frac{h_{0}^{2} \Omega_{\mathrm{b}}}{0.023}\right)^{-1 / 2}\left(\frac{h_{0}^{2} \Omega_{\mathrm{M}}}{0.134}\right)^{1 / 4}\left(\frac{1050}{z_{\text {rec }}}\right)^{3 / 4} \text {. }
$$

There might be slightly different choices for the diffusion scale. For instance it has been noticed in the past that the diffusion scale of the magnetic fields should be related with the induced velocity of Alfvén waves. In this case the diffusion length scale will be slightly smaller than the Silk scale by a factor which is essentially proportional, in my notations, to $\left(B_{\mathrm{L}} / \mathrm{nG}\right)$. In this case the diffusion wave number will be given by [19]

$$
k_{\mathrm{D}} \simeq\left(1.7 \times 10^{2}\right)^{2 / \epsilon}\left(\frac{B_{\mathrm{L}}}{\mathrm{nG}}\right)^{-2 /(\epsilon+2)} h_{0}^{1 /(\epsilon+2)} \mathrm{Mpc}^{-1} .
$$

Figure 3 illustrates the situation described by Eq. (4.12). It is clear that the patterns of the different thermal histories remain the same. However, the bound is improved by roughly 1 order of magnitude.

\section{CONCLUDING REMARKS}

There are various lessons that can be drawn from the exercise reported in this paper. The main question I addressed has been the possible influence of slight variations in the thermal history of the Universe on the curvature perturbations induced by a magnetic field present for typical scales that are larger than the Hubble radius at recombination. Various inflationary mechanisms for the generation of cosmic magnetic fields fall in this situation. It has been found that, indeed, the thermal history of the Universe may either suppress or increase the relative weight of magnetized curvature perturbations. The suppression occurs when, during the dynamical evolution, there is a sort of destructive interference. This phenomenon takes place, amusingly enough, when there was a phase, in the life of the Universe, when the rate of expansion was slower than the one of radiation. This kind of evolution must occur prior to the onset of big-bang nucleosynthesis and explicit examples have been provided. Also the opposite phenomenon can be realized, i.e. a sort of constructive interference when the overall contribution of the magnetized curvature perturbations gets enhanced in comparison with the adiabatic contribution present at the end of in- flation. This situation takes place if the reheating phase is sufficiently prolonged in comparison with the sudden reheating approximation.

These considerations can certainly be developed along different directions. It is interesting to remark that the nature of the bounds on the magnetic field intensity may change quantitatively and qualitatively. Different thermal histories imply that the constraints on the magnetic field intensities may vary, generally speaking, of 1 order of magnitude. In the case when the Universe contains after inflation (but before radiation) a stiff phase the present value of the smoothed magnetic field must be qualitatively ${ }^{10} B_{\mathrm{L}}<1.5 \times 10^{-7} \mathrm{G}$ while in the context of prolonged reheating it must be as small as $1.6 \times 10^{-8} \mathrm{G}$.

The presented considerations are not totally insensitive on the estimate of the diffusive wave number. In particular, it occurs that while experimental data favor a slightly red spectrum of curvature perturbations (i.e. an adiabatic spectral index slightly smaller than 1 ), the magnetized contribution is typically nearly scale invariant but slightly blue. In this situation the most constraining wave numbers are the ones close to diffusion scale. If the diffusivity length scale is slightly smaller than the Silk scale, then the overall bounds on the smoothed magnetic field intensity range, depending on the details of the specific thermal history, from $B_{\mathrm{L}}<\mathrm{nG}$ to $B_{\mathrm{L}}<0.1 \mathrm{nG}$. It can be discussed if the Alfvén velocity should enter the thermal diffusivity scale when the magnetic field is fully inhomogeneous. Indeed Alfvén waves are only excited when there is a background magnetic field in the game. This is not the case when the magnetic field does not break spatial isotropy which is the case investigated in the present paper and which is also, in my opinion, the most realistic one (see the introduction). With these caveats, however, it is important to notice that different thermal histories may either relax or improve the bounds on the magnetic field intensity by 1 order of magnitude.

\footnotetext{
${ }^{10}$ It should be stressed, as discussed in Sec. IV, that $B_{\mathrm{L}}$ is the intensity of the magnetic field at recombination redshifted to the present epoch. This field is by no means equal to the present magnetic field. So the bounds on $B_{\mathrm{L}}$ are, really and truly, bounds on a primordial magnetized background. The present magnetic field of galaxies and clusters is certainly related to $B_{\mathrm{L}}$. In the simplest case, compressional amplification may turn a $0.1 \mathrm{nG}$ field (coherent over a comoving scale of the order of the Mpc) into the $\mu \mathrm{G}$ field observed in galaxies.
}

[1] Ya. B. Zeldovich, A. A. Ruzmaikin, and D. D. Sokoloff, Magnetic Fields in Astrophysics (Gordon and Breach Science, New York, 1983).
[2] E. Battaner and E. Florido, Fundam. Cosm. Phys. 21, 1 (2000).

[3] P. P. Kronberg, Rep. Prog. Phys. 57, 325 (1994). 
[4] M. Giovannini, Int. J. Mod. Phys. D 13, 391 (2004).

[5] B. Ratra, Astrophys. J. 391, L1 (1992).

[6] M. S. Turner and L. M. Widrow, Phys. Rev. D 37, 2743 (1988).

[7] M. Gasperini, M. Giovannini, and G. Veneziano, Phys. Rev. Lett. 75, 3796 (1995).

[8] M. Giovannini, Phys. Rev. D 62, 123505 (2000).

[9] K. E. Kunze, Phys. Lett. B 623, 1 (2005).

[10] Ya. Zeldovich and I. Novikov, The Structure and Evolution of the Universe (Chicago University Press, Chicago, 1971), Vol. 2; Ya. Zeldovich, Sov. Phys. JETP 21, 656 (1965); K. Thorne, Astrophys. J. 148, 51 (1967); S. Hawking and R. J. Tayler, Nature (London) 209, 1278 (1966); J. Barrow, Mon. Not. R. Astron. Soc. 175, 359 (1976); Phys. Rev. D 55, 7451 (1997).

[11] A. Hajian and T. Souradeep, Phys. Rev. D 74, 123521 (2006); T. Souradeep, A. Hajian, and S. Basak, New Astron. Rev. 50, 889 (2006); P. D. Naselsky, L. Y. Chiang, P. Olesen, and O. V. Verkhodanov, Astrophys. J. 615, 45 (2004); G. Chen, P. Mukherjee, T. Kahniashvili, B. Ratra, and Y. Wang, Astrophys. J. 611, 655 (2004).

[12] M. Giovannini, Phys. Rev. D 73, 101302 (2006).

[13] M. Giovannini, Phys. Rev. D 74, 063002 (2006).

[14] M. Giovannini, Report No. CERN-PH-TH/2007-110.

[15] J. D. Barrow, R. Maartens, and C. G. Tsagas, Phys. Rep. 449, 131 (2007).

[16] T. Kahniashvili and B. Ratra, Phys. Rev. D 75, 023002 (2007).

[17] K. Subramanian and J. D. Barrow, Mon. Not. R. Astron. Soc. 335, L57 (2002).

[18] K. Subramanian, T. R. Seshadri and J.D. Barrow, Mon. Not. R. Astron. Soc. 344, L31 (2003).

[19] A. Mack, T. Kahniashvili, and A. Kosowsky, Phys. Rev. D 65, 123004 (2002).

[20] K. Subramanian, arXiv:astro-ph/0601570.

[21] M. Giovannini, Classical Quantum Gravity 23, R1 (2006).

[22] D. N. Spergel et al. (WMAP Collaboration), Astrophys. J. Suppl. Ser. 170, 377 (2007).

[23] L. Page et al. (WMAP Collaboration), Astrophys. J. Suppl. Ser. 170, 335 (2007).

[24] H. V. Peiris et al. (WMAP Collaboration), Astrophys. J. Suppl. Ser. 148, 213 (2003).

[25] D. N. Spergel et al. (WMAP Collaboration), Astrophys. J. Suppl. Ser. 148, 175 (2003).
[26] C. L. Bennett et al. (WMAP Collaboration), Astrophys. J. Suppl. Ser. 148, 1 (2003).

[27] S. Cole et al. (2dFGRS Collaboration), Mon. Not. R. Astron. Soc. 362, 505 (2005).

[28] T. E. Montroy et al., Astrophys. J. 647, 813 (2006).

[29] C. L. Kuo et al. (ACBAR Collaboration), Astrophys. J. 600, 32 (2004).

[30] A. C. S. Readhead et al., Astrophys. J. 609, 498 (2004).

[31] C. Dickinson et al., Mon. Not. R. Astron. Soc. 353, 732 (2004).

[32] W. L. Freedman et al., Astrophys. J. 553, 47 (2001).

[33] D. J. Eisenstein et al. (SDSS Collaboration), Astrophys. J. 633, 560 (2005).

[34] M. Tegmark et al. (SDSS Collaboration), Astrophys. J. 606, 702 (2004).

[35] E. Semboloni et al., arXiv:astro-ph/0511090.

[36] H. Hoekstra et al., Astrophys. J. 647, 116 (2006).

[37] P. Astier et al. (SNLS Collaboration), Astron. Astrophys. 447, 31 (2006).

[38] A. G. Riess et al. (Supernova Search Team Collaboration), Astrophys. J. 607, 665 (2004).

[39] B. J. Barris et al., Astrophys. J. 602, 571 (2004).

[40] M. Giovannini, Phys. Rev. D 64, 061301 (2001).

[41] M. Giovannini (unpublished).

[42] M. Giovannini, Phys. Rev. D 56, 3198 (1997).

[43] J. M. Bardeen, Phys. Rev. D 22, 1882 (1980).

[44] J. Bardeen, P. Steinhardt, and M. Turner, Phys. Rev. D 28, 679 (1983).

[45] R. Brandenberger, R. Kahn, and W. Press, Phys. Rev. D 28, 1809 (1983).

[46] D. H. Lyth, Phys. Rev. D 31, 1792 (1985).

[47] W. Hu and N. Sugiyama, Astrophys. J. 444, 489 (1995).

[48] W. Hu and N. Sugiyama, Astrophys. J. 471, 542 (1996).

[49] H. Kodama and M. Sasaki, Prog. Theor. Phys. Suppl. 78, 1 (1984).

[50] M. Giovannini, Phys. Rev. D 58, 083504 (1998).

[51] L. P. Grishchuk, Ann. N.Y. Acad. Sci. 302, 439 (1977).

[52] M. Giovannini, Phys. Rev. D 60, 123511 (1999).

[53] M. Giovannini, Classical Quantum Gravity 16, 2905 (1999).

[54] V. Sahni, M. Sami, and T. Souradeep, Phys. Rev. D 65, 023518 (2001).

[55] P. J.E. Peebles and A. Vilenkin, Phys. Rev. D 59, 063505 (1999).

[56] L. H. Ford, Phys. Rev. D 35, 2955 (1987). 